\title{
Determinación de la digestibilidad de la proteína, aminoácidos y energía de canola integral en cerdos en crecimiento
}

\section{Determination of the digestibility of the protein, amino acids and energy of full fat canola in growing pigs}

\author{
Gerardo Mariscal-Landín*, Erika Ramírez Rodrígueza
}

\begin{abstract}
RESUMEN
Para evaluar el uso de canola integral en la alimentación de cerdos se realizaron dos experimentos. En el primero se evaluó la digestibilidad aparente y estandarizada a nivel ileal (DAI y DEI) de la materia seca (MS), proteína (PC), aminoácidos (AA) y energía (E); en el segundo se evaluó la digestibilidad aparente fecal (DAF) de la PC y E. En ambos experimentos se evaluaron dos dietas de canola integral, una con canola integral molida más almidón de maíz (CIMAM) y otra con canola integral y sorgo molidos juntos (CISM). Los resultados muestran que la DAI de la MS (71.4), PC $(54.9)$ y $E(67.6)$ fue similar $(P>0.10)$ en ambas dietas. La DAI y DEI difirieron en los aminoácidos alanina y leucina $(P<0.001)$, fenilalanina, ácido glutámico, histidina y valina $(P<0.01)$, y la DEI de serina $(P<0.05)$. La mayor digestibilidad se observó en la dieta CISM. La DAF de la MS y PC $(P<0.001)$ y de E $(P<0.01)$ fueron menores en la dieta CISM. Se concluye que la DAI de la proteína y aminoácidos, así como la DAF de la energía, son bajas en la canola integral molida, lo que reduce la posibilidad de incluirla en dietas para cerdos. Sin embargo, la molienda de canola integral junto con el sorgo mejoró la digestibilidad aparente de los aminoácidos de la mezcla, lo que permitiría utilizar esa mezcla como un ingrediente en la formulación de raciones.
\end{abstract}

PALABRAS CLAVE: Canola integral, Energía, Aminoácidos, Digestibilidad, Cerdos.

\begin{abstract}
Two experiments were carried out to evaluate in pigs the use of full-fat canola. In the first one apparent and standardized ileal digestibility (AID and SID) of dry matter (DM), crude protein (CP), amino acids (AA) and energy (E) were evaluated in twelve pigs. In the second one apparent fecal digestibility (AFD) of CP and E were evaluated in eight pigs. In both experiments, full-fat canola (FFC) was used to prepare two diets, a full-fat canola milled and corn starch diet (FFCM-CS), and another with full-fat canola and sorghum milled together (FFCSM). The results show that AID of DM (71.4), CP (54.9) and E (67.6) was similar ( $P>0.10)$ between the two diets. SID and AID differ in alanine and leucine $(P<0.001)$, phenylalanine, glutamic acid, histidine and valine $(P<0.01)$; and The SID of serine $(P<0.05)$. In all cases, the higher digestibility was observed in the FFCSM diet. The AFD of DM and CP $(P<0.001)$, and E were lower $(P<0.01)$ in FFCSM diet than in FFCM-CS diet. It is concluded that AID and SID of protein and amino acids, as well as AFD of energy, are low in full-fat canola, reducing the possibility of its inclusion in diets for pigs. However, the mix of full-fat canola and sorghum milled together improved apparent ileal digestibility of amino acids allowing its use as an ingredient in the formulation of rations.
\end{abstract}

KEY WORDS: Full fat canola, Energy, Amino acids, Digestibility, Pigs.

\footnotetext{
Recibido el 14 de diciembre de 2015. Aceptado el 6 de julio de 2016.

a Centro Nacional de Investigación en Fisiología Animal - INIFAP, km 1 Carretera a Colón, 76280, Ajuchitlán Querétaro, México.

* Autor de Correspondencia: mariscal.gerardo@inifap.gob.mx.
} 
La semilla de canola es rica en aceite y proteína ${ }^{(1,2)}$, sin embargo también es rica en fibra detergente neutra ${ }^{(1,2)}$ y contiene glucosinolatos ${ }^{(3)}$, los cuales pueden afectar la utilización de los nutrientes y el consumo de alimento. Estos efectos antinutricionales son debidos a que la semilla de canola contiene la enzima mirosinasa, la cual es activada al sufrir un daño la semilla. La mirosinasa activa separa la glucosa de los glucosinolatos y produce los compuestos tóxicos isotiocianatos, oxazolidinethiones(4). Durante el proceso de extracción del aceite, esta enzima se inactiva, ya que es una enzima termolábil(5), por lo que la pasta de canola no la contiene. Sin embargo se desconoce si el uso de canola integral, la cual no es sometida a un tratamiento térmico previo pudiera afectar el consumo de alimento y la digestibilidad de la proteína. Además de que la información sobre su valor nutritivo como alimento para cerdos es escasa $^{(6)}$, pero necesaria para saber si su uso en las dietas de cerdos afecta su consumo y la digestibilidad de los nutrientes.

Por lo anterior, los objetivos de este estudio fueron evaluar la digestibilidad ileal y fecal de la energía de la canola integral en cerdos en crecimiento; así como la digestibilidad estandarizada ileal (DEI) de sus aminoácidos. Para alcanzar el objetivo se realizaron dos experimentos en la unidad metabólica de la granja experimental del CENIDFisiología localizada en Ajuchitlán, Qro. El protocolo fue revisado por el Comité "Grupo Colegiado Científico Técnico" el cual avaló que el manejo empleado en los animales experimentales respetara los lineamientos de la Norma Oficial Mexicana para la producción, cuidado y uso de los animales de laboratorio(7) así como los de la "International Guiding Principles for Biomedical Research Involving Animals".

En el estudio se utilizó sorgo y canola integral; la canola integral se molió sola o en combinación con el sorgo en la siguiente proporción (75 \% sorgo, $25 \%$ canola integral) en un molino de martillos. Con los productos molidos se crearon dos tratamientos (Cuadro 1): canola integral molida-almidón de maíz (CIM-AM), canola integral-sorgo molidos juntos (CISM). A ambas dietas se les agregó sacarosa a razón de $50 \mathrm{~g} / \mathrm{kg}$ para incrementar su palatabilidad.
Las vitaminas y minerales se adicionaron para proporcionar 0 exceder los requerimientos recomendados por el $\mathrm{NRC}^{(8)}$. La digestibilidad de las dietas se evaluó por medio de la adición de óxido de titanio a razón de $3 \mathrm{~g} / \mathrm{kg}$ de alimento.

Durante el periodo experimental los cerdos se alimentaron dos veces al día (0800 y 1800 h); la cantidad de alimento ofrecido diariamente aportó 2.5 veces su requerimiento de energía digestible (ED) de mantenimiento, el cual se estimó en 110 $\mathrm{kcal}$ de ED/kg de PV ${ }^{0.75}$, INRA ${ }^{(9)}$.

En el experimento de digestibilidad ileal se usaron un total de 12 cerdos machos castrados de la línea 'Genetiporc" (Fertilis 20 x G Performance) con un peso de $35.0 \pm 1.5 \mathrm{~kg}$ al momento de la cirugía; a los cuales se les implantó una cánula " $T$ " en el íleon terminal(10). Después de la cirugía los cerdos se alojaron individualmente en jaulas metabólicas localizadas en una sala con temperatura controlada a $19 \pm 2{ }^{\circ} \mathrm{C}$. El periodo postoperatorio duró 21 días, durante los cuales los cerdos consumieron una dieta de crecimiento con $160 \mathrm{~g} / \mathrm{kg}$ de PC, proporcionada dos veces al día (0800 y 1800 h). La cantidad de alimento ofrecido se incrementó diariamente hasta que los cerdos alcanzaron su consumo previo a la cirugía. El agua se proporcionó a libertad a través de un bebedero de chupón localizado en una pared de la jaula metabólica.

El periodo experimental tuvo una duración de siete días (cinco de adaptación a la dieta y dos de colecta ileal). La digesta ileal se colectó en bolsas de plástico (de $11 \mathrm{~cm}$ de largo $\times 5 \mathrm{~cm}$ de ancho), a las cuales se les agregaron $10 \mathrm{ml}$ de una solución de $\mathrm{HCl} 0.2 \mathrm{M}$ con el objeto de bloquear toda actividad bacteriana. Las bolsas se fijaron a la cánula con una liga a las $0800 \mathrm{~h}$ del día uno y se colectó la digesta ileal durante $10 \mathrm{~h}$ continuas los dos días de muestreo. Al llenar la digesta ileal, la bolsa fijada en la cánula se transfirió a un contenedor para proceder inmediatamente a congelarla a $-20{ }^{\circ} \mathrm{C}$ hasta su liofilización.

En el experimento de digestibilidad fecal se usaron un total de ocho cerdos machos castrados de la línea 'Genetiporc" (Fertilis 20 x G Performance) con un peso promedio de $35.0 \pm 1.5 \mathrm{~kg}$; los cerdos se alojaron en corraletas individuales, el agua se 
Cuadro 1. Materias primas y dietas experimentales. Composición química en porcentaje

\begin{tabular}{|c|c|c|c|c|}
\hline \multirow[b]{2}{*}{ Ingrediente } & \multicolumn{2}{|c|}{ Materias primas } & \multicolumn{2}{|c|}{ Dietas experimentales } \\
\hline & $\begin{array}{l}\text { Canola } \\
\text { integral }\end{array}$ & Sorgo & $\begin{array}{l}\text { Canola* } \\
\text { almidón }\end{array}$ & $\begin{array}{c}\text { Canola } \\
\text { sorgo }\end{array}$ \\
\hline \multicolumn{5}{|l|}{ Composición químicaa } \\
\hline Proteína, \% & 21.0 & 8.0 & 10.1 & 10.1 \\
\hline Energía, Kcal/kg & 6870 & 3200 & 5083 & 4792 \\
\hline Acido aspártico & 1.80 & 0.42 & 0.86 & 0.69 \\
\hline Ácido glutámico & 4.76 & 1.97 & 2.28 & 2.40 \\
\hline Serina & 1.16 & 0.35 & 0.56 & 0.50 \\
\hline Histidina & 0.77 & 0.37 & 0.37 & 0.43 \\
\hline Glicina & 0.99 & 0.15 & 0.48 & 0.33 \\
\hline Treonina & 1.69 & 0.40 & 0.81 & 0.65 \\
\hline Arginina & 1.56 & 0.28 & 0.75 & 0.54 \\
\hline Alanina & 1.23 & 1.31 & 0.59 & 1.16 \\
\hline Tirosina & 0.76 & 0.33 & 0.36 & 0.39 \\
\hline Valina & 1.13 & 0.43 & 0.54 & 0.54 \\
\hline Fenilanina & 1.11 & 0.32 & 0.53 & 0.46 \\
\hline Isoleucina & 0.79 & 0.29 & 0.38 & 0.38 \\
\hline Leucina & 1.67 & 1.02 & 0.80 & 1.06 \\
\hline Lisina & 2.00 & 0.26 & 0.96 & 0.62 \\
\hline \multicolumn{5}{|c|}{ Composición porcentual de las dietas experimentales } \\
\hline $100 \%$ canola & & & 48.00 & \\
\hline $25 \%$ canola-75\%sorgo & & & & 90.00 \\
\hline Almidón de maíz & & & 44.24 & 2.24 \\
\hline Azúcar & & & 5.00 & 5.00 \\
\hline Carbonato de calcio & & & 0.90 & 0.90 \\
\hline Ortofosfato & & & 0.88 & 0.88 \\
\hline Sal & & & 0.50 & 0.50 \\
\hline Vitaminas ${ }^{A}$ & & & 0.16 & 0.16 \\
\hline Minerales ${ }^{B}$ & & & 0.07 & 0.07 \\
\hline Titanio & & & 0.25 & 0.25 \\
\hline \multicolumn{5}{|c|}{${ }^{* \star}$ Dieta canola integral sorgo molidos. } \\
\hline \multicolumn{5}{|c|}{ a En base tal y como se ofrece. } \\
\hline \multicolumn{5}{|c|}{$\begin{array}{l}\text { A Proporciona por kilo de alimento: Vitamina A } 6600 \mathrm{UI}, \text { D } 660 \mathrm{UI}, \text { E } 100 \mathrm{UI} \text {, colina } 350 \mathrm{mg} \text {, niacina } 54 \text { mg, ácido pantoténico } 13.15 \mathrm{mg} \text {, } \\
\text { riboflavina } 2.2 \mathrm{mg}, \text { B12 } 36 \mathrm{~g} \text {. }\end{array}$} \\
\hline B Proporciona por kilo de alim & & & & \\
\hline
\end{tabular}

proporcionó a libertad a través de un bebedero de chupón localizado en una pared de la corraleta individual. El periodo experimental tuvo una duración de 10 días (cinco de adaptación a la dieta y cinco de colecta de excremento).

Las muestras de digesta ileal se liofilizaron y las de excremento se secaron a $55^{\circ} \mathrm{C}$ durante $48 \mathrm{~h}$. La digesta ileal liofilizada, el excremento seco y las muestras de alimento, se molieron a través de una malla de $0.5 \mathrm{~mm}$ en un molino de laboratorio (Arthur $\mathrm{H}$. Thomas Co. Philadelphia, PA). Los siguientes análisis se realizaron en las dietas experimentales y en las muestras de digesta ileal y excremento: materia seca (MS) y proteína (PC) de acuerdo a los métodos 934.01 y 976.05 del $A O A C^{(11)}$, óxido de titanio según Myers $^{(12)}$. La preparación de las 
muestras para la determinación de aminoácidos (AA) se realizó según el método 994.12 del $A O A C^{(11)}$, el cual consiste en hidrolizar las muestras a $110^{\circ} \mathrm{C}$ durante $24 \mathrm{~h}$ en $\mathrm{HCl} 6 \mathrm{M}$. Los análisis de $\mathrm{AA}$ se realizaron por medio de cromatografía en fase reversa según el método descrito por Henderson et $a^{(13)}$ en un HPLC de marca Hewlett Packard, modelo 1100. Los análisis de energía se realizaron por medio de una bomba calorimétrica adiabática (modelo 1281, Parr, Moline, IL).

Los cálculos para estimar los coeficientes de digestibilidad aparente a nivel ileal (CDAI) y fecal (CDAF) de la MS, PC y E; y los CDAI y los coeficientes de digestibilidad aparente a nivel ileal (CDAI) de los aminoácidos en las dietas experimentales se realizaron empleando la siguiente ecuación ${ }^{(14)}$

$$
C D A I \text { o } C D A F=1-[(I D \times A F) /(A D \times I F)]
$$

Donde $C D A I$ es el coeficiente de digestibilidad aparente ileal o el $C D A F$ coeficiente de digestibilidad aparente fecal de un nutrimento en la dieta, $I_{D}$ es la concentración del indicador en la dieta $(\mathrm{mg} / \mathrm{kg}$ de $\mathrm{MS}), A_{F}$ es la concentración del nutrimento en la digesta ileal (mg/kg de MS), $A_{D}$ es la concentración del nutrimento en la dieta $\left(\mathrm{mg} / \mathrm{kg}\right.$ de MS), $I_{F}$ es la concentración del indicador en la digesta ileal (mg/kg de MS).

Para estimar los CDEI de los aminoácidos se utilizó la fórmula propuesta por Mariscal-Landín ${ }^{(15)}$.

$$
C D E I=C D A I+(\text { endógeno } / \text { consumido })
$$

Donde $C D E I$ es el coeficiente de digestibilidad estandarizada ileal de un nutrimento, $C D A I$ es el coeficiente de digestibilidad aparente ileal de un nutrimento, endógeno es la cantidad endógena excretada del nutrimento en $\mathrm{mg} / \mathrm{kg}$ de materia seca consumida, en el cálculo se utilizó el endógeno reportado por Mariscal-Landín y Reis de Souza ${ }^{(16)}$, consumido es la cantidad de nutrimento consumido en $\mathrm{mg} / \mathrm{kg}$ de materia seca consumida.

Los datos de los dos experimentos se analizaron con un diseño completamente al azar ${ }^{(17)}$ empleando

Cuadro 2. Digestibilidad aparente a nivel lleal de la proteína y de los aminoácidos

\begin{tabular}{lcccc}
\hline & Canola-almidón* & Canola-sorgo* & EEM & $P$ \\
\hline Materia seca & 71.5 & 71.4 & 0.53 & 0.85 \\
Proteína cruda & 53.8 & 55.9 & 1.46 & 0.32 \\
Energía & 67.6 & 67.6 & 2.33 & 0.99 \\
Alanina & 53.8 & 77.2 & 1.55 & 0.001 \\
Arginina & 59.8 & 51.9 & 3.65 & 0.16 \\
Aspártico & 50.9 & 52.3 & 1.59 & 0.55 \\
Fenilanina & 66.2 & 78.4 & 2.61 & 0.01 \\
Glicina & 51.2 & 42.9 & 2.86 & 0.10 \\
Glutámico & 65.0 & 74.3 & 1.65 & 0.01 \\
Histidina & 75.6 & 82.7 & 1.38 & 0.01 \\
Isoleucina & 49.5 & 55.3 & 2.91 & 0.20 \\
Lisina & 71.5 & 65.5 & 2.45 & 0.12 \\
Leucina & 60.8 & 75.1 & 1.35 & 0.001 \\
Serina & 50.0 & 55.9 & 1.97 & 0.10 \\
Treonina & 44.7 & 49.5 & 2.93 & 0.28 \\
Tirosina & 37.8 & 47.0 & 3.56 & 0.10 \\
Valina & 57.5 & 66.6 & 1.89 & 0.01 \\
\hline * Dieta canola integral molida - almidón de maíz. & & & \\
** Dieta canola integral sorgo molidos. & & & & \\
EEM= error estándar de la media. & & & \\
$P=$ nivel de probabilidad de la diferencia entre los tratamientos.. & & & \\
& & & &
\end{tabular}


el procedimiento GLM del paquete estadístico SAS ${ }^{(18)}$.

Los resultados muestran que la DAI de la MS (71.5 vs 71.4) de la PC (53.8 vs 55.9) y de la E (67.6 vs 67.6) fue similar ( $P>0.10$ ) para ambas dietas CIM-AM y CISM respectivamente (Cuadro 2). Los aminoácidos en los que su DAI (Cuadro 2) y su DEI (Cuadro 3) difirió entre dietas fueron: alanina y leucina $(P<0.001)$, fenilalanina, ácido glutámico, histidina y valina $(P<0.01)$; y la DEI de serina $(P<0.05)$. La mayor digestibilidad se observó siempre en la dieta CISM (Cuadro 2).
Digestibilidad fecal. Los resultados muestran una disminución $(P<0.001)$ en la DFA de la materia seca, y PC y de la $\mathrm{E}(P<0.01)$ en la dieta CISM (Cuadro 4). La disminución fue de 6.1 unidades (82.4 a 76.3) para la MS, de 19.3 unidades (72.2 a 52.9) para la PC, y de 5.5 unidades (77.7 a 72.2) para la $\mathrm{E}$.

Las principales proteínas de la canola son la napina, cruciferina y la oleosina. Las dos primeras son proteínas de reserva y constituyen el 20 y el $60 \%$ de las proteínas de canola respectivamente. La oleosina es una proteína estructural asociada con

Cuadro 3. Digestibilidad estandarizada a nivel ileal de la proteína y de los aminoácidos

\begin{tabular}{lcccc}
\hline & Canola-almidón & Canola-sorgo** & EEM & $P$ \\
\hline Proteína cruda & 72.3 & 74.3 & 1.46 & 0.35 \\
Alanina & 66.8 & 83.8 & 1.55 & 0.001 \\
Arginina & 73.3 & 70.5 & 3.65 & 0.60 \\
Aspártico & 64.1 & 68.7 & 1.59 & 0.10 \\
Fenilanina & 74.6 & 88.0 & 2.61 & 0.01 \\
Glicina & 76.0 & 78.8 & 2.86 & 0.51 \\
Glutámico & 72.6 & 81.5 & 1.65 & 0.01 \\
Histidina & 80.0 & 86.4 & 1.38 & 0.01 \\
Isoleucina & 66.1 & 72.0 & 2.91 & 0.18 \\
Leucina & 70.2 & 82.2 & 1.35 & 0.001 \\
Lisina & 74.7 & 70.4 & 2.45 & 0.25 \\
Serina & 66.8 & 74.5 & 1.97 & 0.05 \\
Treonina & 54.7 & 61.9 & 2.93 & 0.12 \\
Tirosina & 48.1 & 56.6 & 3.56 & 0.13 \\
Valina & 71.7 & 80.6 & 1.89 & 0.01 \\
\hline
\end{tabular}

" Dieta canola integral molida - almidón de maíz.

* Dieta canola integral sorgo molidos.

$\mathrm{EEM}=$ error estándar de la media.

$P=$ nivel de probabilidad de la diferencia entre los tratamientos.

Cuadro 4. Digestibilidad aparente a nivel fecal

Canola-almidón

Canola-sorgo**

EEM

$P$

\begin{tabular}{lllll}
\hline Materia seca & 82.4 & 76.3 & 0.87 & 0.001 \\
Proteína & 72.2 & 52.9 & 1.61 & 0.001 \\
Energía & 77.7 & 72.2 & 1.19 & 0.01 \\
\hline
\end{tabular}

* Dieta canola integral molida - almidón de maíz.

* Dieta canola integral sorgo molidos.

EEM $=$ error estándar de la media.

$P=$ nivel de probabilidad de la diferencia entre los tratamientos. 
la fracción aceitosa, la cual representa del 2 al $8 \%$ de las proteínas de canola(19). El aceite de canola se caracteriza por su bajo contenido (7\%) de ácidos grasos saturados, su alto contenido en ácido grasos mono-insaturados (MUFA) y poli-insaturados (PUFA), incluyendo $61 \%$ de ácido oleico, $21 \%$ de ácido linoleico y $11 \%$ de ácido alfa linolénico ${ }^{(20)}$. El aceite de canola tiene un punto de fusión entre -10 y $-5{ }^{\circ} \mathrm{C}$ y tiene un índice de per-oxidación de $40^{(21)}$, lo que lo convierte en un aceite susceptible de ser per-oxidado. La semilla de canola también es rica en fibra detergente neutra (FDN) $16 \%$, fibra detergente ácido (FDA) $12 \%$ y lignina ácida $5 \%$, además de que alrededor del $36 \%$ de su proteína está ligada a FDN y el $22 \%$ está ligada a FDA(22).

Existen pocos trabajos que reporten el uso de canola integral en la alimentación del cerdo. Woyengo et $a^{(6)}$ mencionan que al utilizar canola integral hasta un $15 \%$ se disminuyó de manera lineal la eficiencia alimenticia, el peso y rendimiento de la canal y tendió a disminuir la ganancia diaria de peso. Thacker ${ }^{(23)}$ estudió el uso de canola integral y canola integral micronizada, mencionando que el uso de canola integral afectó el consumo de alimento en hembras, pero no en machos castrados, lo que provocó una disminución de las ganancias diarias de peso de las hembras alimentadas con canola integral; el consumo de canola integral no afectó las características de la canal aunque ésta tendió a ser más grasosa. Esos efectos no los observaron con el uso de canola integral micronizada.

La digestibilidad ileal aparente de la proteína de canola integral reportada por Woyengo et $a^{(6)}$ fue mayor a la obtenida en este trabajo (67.8 y $53.8 \%$ respectivamente); y esto se repitió en el caso de la DAI de los aminoácidos reportados por estos mismos investigadores $^{(6)}$ y por otros ${ }^{(24)}$. La baja digestibilidad de la proteína de la semilla de canola observada en este trabajo puede ser debida a que las dietas experimentales contenían solamente $10 \%$ de proteína, y se sabe que niveles bajos de proteína dietaria afectan negativamente la digestibilidad aparente de la proteína y aminoácidos a nivel ileal ${ }^{(14,25,26)}$; ya que la proteína y los aminoácidos endógenos son proporcionalmente más importantes en la digesta ileal disminuyendo la digestibilidad aparente $\mathrm{a}^{(14,25-27)} ;$ y a que dietas con un nivel bajo de proteína provocan una menor actividad de las enzimas: tripsina, amilasa pancreática, quimiotripsina, enterocinasa y dipeptidasa $\mathrm{II}^{(28)}$, lo que pudiera repercutir en una menor digestibilidad de la proteína. Otra posible explicación es que durante el proceso industrial de extracción del aceite, la semilla es calentada a $85{ }^{\circ} \mathrm{C}$, lo que pudiera desnaturalizar algunas proteínas haciéndolas más digestibles como sucede con la pasta de soya(29); además de inhibir la enzima mirosinasa, efecto que no se obtiene con la simple molienda de la semilla de canola; por lo que en la canola integral molida sola o en presencia de sorgo, la enzima mirosinasa pudo estar activa liberando los glucósidos cianogénicos ${ }^{(29)}$. Esto explicaría la mejora observada por Thacker(23) cuando micronizó la canola integral. Finalmente, otra posible causa es la presencia de grasa peroxidada, porque afecta la capacidad de absorción del intestino delgado y modifica la morfología intestinal al alterar el potencial redox a nivel luminal( ${ }^{(30)}$, afectando in vivo la membrana del enterocito, e in vitro la actividad de las enzimas sacarasa, maltasa y fosfatasa alcalina $^{(31)}$. La molienda de la semilla de canola pudo favorecer este efecto porque no se utilizaron antioxidantes durante ese proceso. Esta acción de la grasa es opuesta a la reportada previamente, en la cual se mencionaba que el alto contenido de aceite en las dietas incrementaba la digestibilidad de la proteína dietaria ${ }^{(32)}$, y de los aminoácidos ${ }^{(33)}$; sin embargo, en esos trabajos el aceite adicionado había sido procesado industrialmente, por lo que contenían antioxidantes que prevenían su peroxidación.

Al estimar la digestibilidad estandarizada de la proteína y aminoácidos de las dietas experimentales, se observó en ambas dietas una menor digestibilidad ileal estandarizada que los valores de referencia de pasta de canola ${ }^{(1,2)}$. La mayor digestibilidad de los aminoácidos (alanina, fenilalanina, ácido glutámico, histidina, serina y valina) en la dieta CISM, pudo ser debida a que la presencia del sorgo disminuyó el posible daño a la proteína de canola. Esto concuerda con las mejores digestibilidades reportadas en dietas de canola integral tratada en presencia de otra materia prima (trigo o chícharo) ${ }^{(6,24)}$. 
En lo que respecta a la mayor digestibilidad fecal de la materia seca, proteína y energía en la dieta CIM-AM; esto pudiera explicarse por el menor contenido de fibra en esa dieta, lo cual concuerda con observaciones previas, en las que la inclusión de fibra disminuyó de manera lineal la digestibilidad fecal de esos nutrientes ${ }^{(34)}$.

Se concluye que la digestibilidad aparente a nivel ileal de la proteína y aminoácidos; así como la digestibilidad aparente a nivel fecal de la energía en la canola integral son bajas. Esta baja digestibilidad reduce su posibilidad de inclusión en dietas para cerdos. Sin embargo, la molienda de canola integral junto con sorgo mejoró la digestibilidad de los aminoácidos de la mezcla, lo que permitiría utilizar esa mezcla como un ingrediente más en la formulación de raciones para cerdos.

\section{AGRADECIMIENTOS}

Se agradece al INIFAP por su apoyo financiero al proyecto "Incorporación de las oleaginosas con mayor potencial en México, para la solución de una problemática fundamental en los mercados agrícola, industrial y pecuario". Proyecto SIGI No. 11311419345. Así como a la Química Jasmín Ruíz Jiménez, por los análisis de aminoácidos; a la MVZ Yamily Ramírez por los análisis de laboratorio, y a los MVZ Julio César Baltazar Vázquez y Víctor Balderrama Pérez por el cuidado de los animales.

\section{LITERATURA CITADA}

1. INRA. Tables de composition et de valeur nutritive des matières premières destinées aux animaux d'élevage. Porcs, volailles, bovins, ovins, caprins, lapins, chevaux, poissons. Paris, France: Institut National de la Recherche Agronomique; 2002.

2. NRC. Nutrient requirements of swine: 11th Rev ed. Washington, DC: The National Academies Press; 2012.

3. Gilani GS, Chao WX, Cockell KA. Impact of antinutritional factors in food proteins on the digestibility of protein and the bioavailability of amino acids and on protein quality. Br J Nutr 2012;108:S315S332.

4. Jan T, Erik A, Susanna E, Lars R. Regulation of the wound-induced myrosinase-associated protein transcript in Brassica napus plants. European J Biochem 1997;247(3):963-971.

5. Woyengo TA, Beltranena E, Zijlstra RT. Nonrum Nutr Symp: Controlling feed cost by including alternative ingredients into pig diets: A review. J Anim Sci 2014;92:1293-1305.
6. Woyengo TA, Yánez J, Young MG, Lanz G, Beltranena E, Zijlstra RT. Nutritional value of full-fat green canola seed fed to growingfinishing pigs. J Anim Sci 2014;92(8):3449-3459.

7. Diario Oficial de la Federación. Especificaciones técnicas para la producción, cuidado y uso de los animales de laboratorio. Norma Oficial Mexicana NOM-062-ZOO-1999. Diario Oficial de la Federación 2001 (miércoles 2 de agosto).

8. NRC. Nutrient requirements of swine: 10th Rev Ed. Washington, DC: The National Academy Press; 1998.

9. INRA. L'alimentation des animaux monogastriques: porc, lapin, volailles. Paris, France: Institut National de la Recherche Agronomique; 1984.

10. Reis de Souza TC, Mar BB, Mariscal LG. Canulación de cerdos posdestete para pruebas de digestibilidad ileal: Desarrollo de una metodología. Téc Pecu Méx 2000;38:143-150.

11. AOAC. Official Methods of Analysis. 17th ed. Arlington, VA. USA: Assoc. Offic. Anal. Chem. 2000.

12. Myers WD, Ludden PA, Nayigihugu V, Hess BW. Technical Note: A procedure for the preparation and quantitative analysis of samples for titanium dioxide. J Anim Sci 2004;82:179-183.

13. Henderson JH, Ricker RD, Bidlingmeyer BA, Woodward C. Rapid, accurate and reproducible HPLC analysis of amino acids. Amino acid analysis using Zorbax Eclipse AAA columns and the Agilent 1100 HPLC. Agilent technologies 2000 (Part No.5980-1193E). www.agilent.com/chem/supplies.

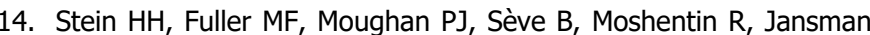
AJM, et al. Deffinition of apparent, true and standardized ileal digestibility of amino acids in pigs. Livest Sci 2007;109:282-285.

15. Mariscal-Landín G. Facteurs de variation de l'utilisation digestive des acides aminés chez le porc. Rennes, France: Université de Rennes; 1992.

16. Mariscal-Landín G, Reis de Souza TC. Endogenous ileal losses of nitrogen and amino acids in pigs and piglets fed graded levels of casein. Arch Anim Nutr 2006;60:454-466.

17. Steel RGD, Torrie JH. Principles and procedures of statistics. A Biometrical approach. 2nd ed. New York: McGraw-Hill; 1980.

18. SAS version 9.2. Statistical Analysis Systems Institute User's guide. Statistical Analysis Systems Institute User's guide. 9.2 ed. Cary NC, USA: SAS Institute Inc.; 2008.

19. Aider M, Barbana C. Canola proteins: composition, extraction, functional properties, bioactivity, applications as a food ingredient and allergenicity - A practical and critical review. Trends Food Sci Technol 2011;22(1):21-39.

20. Lin L, Allemekinders $H$, Dansby A, Campbell L, Durance-Tod S, Berger A, et al. Evidence of health benefits of canola oil. Nutr Rev 2013;71(6):370-385

21. Kerr BJ, Kellner TA, Shurson GC. Characteristics of lipids and their feeding value in swine diets. J Anim Sci Biotechnol 2015;6(1):30.

22. Mustafa AF, Christensen DA, McKinnon JJ, Newkirk R. Effects of stage of processing of canola seed on chemical composition and in vitro protein degradability of canola meal and intermediate products. Can J Anim Sci 2000;80(1):211-214.

23. Thacker PA. Effect of micronization of full-fat canola seed on performance and carcass characteristics of growing-finishing pigs. Anim Feed Sci Technol 1998;71:89-97. 
24. Kiarie $\mathrm{E}$, Nyachoti CM. Ileal digestibility of amino acids in coextruded peas and full fat canola for growing pigs. Anim Feed Sci Technol 2007;139:40-51.

25. Mariscal-Landín G, Reis de Souza TC, Hernández DAA, Escobar GK. Pérdidas endógenas de nitrógeno y aminoácidos en cerdos y su aplicación en la estimación de los coeficientes de digestibilidad ileal de la proteína y aminoácidos de las materias primas. Téc Pecu Méx 2009;47:371-388.

26. Stein $\mathrm{HH}$, Sève $\mathrm{B}$, Fuller MF, Moughan PJ, de Lange CFM. Invited review: Amino acid bioavailability and digestibility in pig feed ingredients: Terminology and application. J Anim Sci 2007;85:172180.

27. Furuya S, Kaji Y. Additivity of the apparent and true digestible amino acid supply in barley, maize, wheat or soya bean based diets for growing pigs. Anim Feed Sci Technol 1991;32:321331.

28. Liuqin $\mathrm{H}$, Li W, Zhiqi $\mathrm{X}$, Tiejun L, Kang Y, Zhijie C, et al. Low-protein diets affect ileal amino acid digestibility and gene expression of digestive enzymes in growing and finishing pigs. Amino Acids 2015;48:21-30.
29. Fan MZ, Sauer WC, de Lange CFM. Amino acid digestibility in soybean meal, extruded soybean and full-fat canola for earlyweaned pigs. Anim Feed Sci Technol 1995;52:189-203.

30. Rosero DS, Odle J, Moeser AJ, Boyd RD, van Heugten E. Peroxidised dietary lipids impair intestinal function and morphology of the small intestine villi of nursery pigs in a dose-dependent manner. $\mathrm{Br}$ J Nutr 2015;114:1985-1992.

31. Kanazawa K, Ashida H, Minamoto S, Danno G-i, Natake M. The effects of orally administered linoleic acid and its autoxidation products on intestinal mucosa in rat. J Nutr Sci Vitaminol 1988;34:363-373.

32. Lowrey RS, Pond WG, Loosli JK, Maner JH. Effect of dietary fat level on apparent nutrient digestibility by growing swine. J Anim Sci 1962;21:746-750.

33. Li S, Sauer WC. The effect of dietary fat content on amino acid digestibility in young pigs. J Anim Sci 1994;72:1737-1743.

34. Mariscal-Landín G, Reis de Souza TC, Ramírez RE. Effects of corn gluten feed inclusion at graded levels in a corn-soybean diet on the ileal and fecal digestibility of growing pigs. J Anim Sci Biotechnol 2014;5:40. 